\title{
Uso de aromaterapia en gestantes: una revisión de la literatura
}

\section{Use of aromatherapy in pregnant women: a review of the literature}

\section{Uso de aromaterapia em mulheres grávidas: uma revisão de literatura}

\author{
Ariadna María Angarita-Navarro ${ }^{1}$ \\ Paula Daniela Casas-Cárdenas ${ }^{2}$ \\ Jineth Paola López-Aguirre ${ }^{3}$
}

\section{Resumen}

Introducción: Algunas mujeres, durante su gestación pueden padecer de sintomatologías molestas que interfieren con su cotidianidad como: náuseas, vómitos, dolores lumbares y fluctuaciones emocionales. Tales molestias podrían manejarse con técnicas como la aromaterapia, ofreciéndoles bienestar, sin efectos adversos para el binomio madre e hijo. El objetivo de este artículo es identificar los beneficios que produce la aromaterapia en la gestación, mediante una revisión documental. Metodología: Se realizó una búsqueda de artículos científicos en bases de datos como: Science direct, google academic, EBSCO, Pubmed, Gale Power Search, BASE (Bielefeld academic search engine), Proquest; utilizando los DECS Aromatherapy, Pregnancy, Lavandula, Citrus, Jengibre y Rosa en inglés y español. Además, se usó el conector booleano AND. De esta búsqueda se encontraron 50 artículos de investigación que cumplían con el objetivo propuesto. Resultados: se identificó la utilización de la aromaterapia como recurso para evitar las náuseas, los vómitos y el dolor; también mejora el sueño y disminuye la fatiga. Conclusiones: Se determina que los aceites esenciales usados en los estudios fueron eficaces porque redujeron los síntomas molestos en la mayoría de las gestantes participantes en los estudios analizados.

Palabras clave: Aromaterapia, atención de enfermería, embarazo, lavanda, citrus, jengibre y rosa.

\footnotetext{
Abstract

Introduction: Some women during pregnancy may suffer from uncomfortable symptoms that interfere with their daily life, such as nausea, vomiting, back pain, and emotional fluctuations. Such discomfort could be managed with techniques such as aromatherapy, tending to offer well-being without possible adverse effects for the mother and child. The objective of this article is to identify the benefits of aromatherapy in pregnancy through a documentary review. Methodology:A search of scientific articles was carried out in databases such as: Science direct, google academic, EBSCO, Pubmed, Gale Power Search, BASE (Bielefeld academic search engine), Proquest; using the DECS Aromatherapy, Pregnancy, Lavandula, Citrus, Ginger and Rosa in English and Spanish, using the Boolean connector AND, from this search 50 research articles were obtained that fulfilled the proposed objective. Results: the use of aromatherapy was identified as a resource for nausea, vomiting and pain, in addition to achieving improved sleep and decreased fatigue. Conclusions: It was determined then that the essential oils used in the studies were effective in reducing bothersome symptoms in most
}

\begin{abstract}
Autor de correspondencia*
${ }^{1 *}$ Enfermera, Especialista en Salud Ocupacional, Magíster en Enfermería. Estudiante de Doctorado en Enfermería. Docente, Fundación Universitaria del Área Andina. Bogotá, Colombia. Correo: aangarita7@areandina.edu.co (i) 0000-0001-6717-4871

2 Estudiante de Enfermería. Pregrado. Fundación Universitaria del Área Andina. Bogotá, Colombia. Correo: pcasas3@estudiantes.areandina.edu.co. (D) 0000-0003-2971-4958

3 Estudiante de Enfermería. Pregrado. Fundación Universitaria del Area Andina. Bogotá, Colombia. Correo: jlopez262@estudiantes.areandina.edu. co (D) 0000-0001-7481-8268
\end{abstract}

Recibido: 13 agosto 2021

Aprobado: 28 octubre 2021

Para citar este artículo

Angarita-Navarro AM, Casas-Cárdenas PD, López-Aguirre JP. Uso de aromaterapia en gestantes: una revisión de la literatura. Rev. cienc. cuidad. 2022; 19(1):107-118, https://doi. org/10.22463/17949831.3114

(C) Universidad Francisco de Paula Santander. Este es un artículo bajo la licencia CC-BY-NC-ND 
Introduction: Some women during pregnancy may suffer from uncomfortable symptoms that interfere with their daily life, such as nausea, vomiting, back pain, and emotional fluctuations. Such discomfort could be managed with techniques such as aromatherapy, tending to offer well-being without possible adverse effects for the mother and child. The objective of this article is to identify the benefits of aromatherapy in pregnancy through a documentary review. Methodology:A search of scientific articles was carried out in databases such as: Science direct, google academic, EBSCO, Pubmed, Gale Power Search, BASE (Bielefeld academic search engine), Proquest; using the DECS Aromatherapy, Pregnancy, Lavandula, Citrus, Ginger and Rosa in English and Spanish, using the Boolean connector AND, from this search 50 research articles were obtained that fulfilled the proposed objective. Results: the use of aromatherapy was identified as a resource for nausea, vomiting and pain, in addition to achieving improved sleep and decreased fatigue. Conclusions: It was determined then that the essential oils used in the studies were effective in reducing bothersome symptoms in most of the pregnant women participating in the studies analyzed.

Key words: Aromatherapy, Prenatal Care, Nursing Care, Pregnancy, Lavender, Citrus, Ginger and Rose

\section{Resumo}

Introdução: Algumas mulheres durante a gravidez podem sofrer de sintomas desconfortáveis que interferem em sua vida diária, tais como náuseas, vômitos, dores nas costas e flutuações emocionais. Tal desconforto poderia ser administrado com técnicas como a aromaterapia, visando oferecer bem-estar sem possíveis efeitos adversos para a mãe e o filho. O objetivo deste artigo é identificar os benefícios da aromaterapia na gravidez através de uma revisão documental. Metodologia: Uma pesquisa de artigos científicos foi realizada em bancos de dados como: Science direct, Google academic, EBSCO, Pubmed, Gale Power Search, BASE (motor de pesquisa acadêmica de Bielefeld), Proquest; utilizando o DECS Aromatherapy, Pregnancy, Lavandula, Citrus, Jengibre e Rosa em inglês e espanhol, utilizando o conector Boolean AND, a partir desta pesquisa foram obtidos 50 artigos de pesquisa que cumpriram o objetivo proposto. Resultados: o uso da aromaterapia foi identificado como um remédio para náuseas, vômitos e dores, bem como para melhorar o sono e reduzir a fadiga. Conclusões: Foi determinado que os óleos essenciais utilizados nos estudos foram eficazes na redução de sintomas incômodos na maioria das mulheres grávidas que participaram dos estudos analisados.

Palavra-chave: Aromaterapia, Cuidados pré-natais, Enfermagem, Gravidez, Lavanda, Citrinos, Gengibre e Rosa

\section{Introducción}

Durante la gestación ocurren cambios anatomo-fisiológicos y psicológicos que son absolutamente normales, tales como: cambios a nivel respiratorio, cardiovascular, hematológico, renal y gastrointestinales; estas alteraciones se generan por aumento y aparición de algunas hormonas (1), por crecimiento y modificación anatómica de algunos órganos, y cambios en la funcionalidad de estos. Estas transformaciones alteran el bienestar en la mujer por la presencia de signos y síntomas como: las náuseas, la pirosis, el vómito, mareos, los dolores lumbares por aumento del tamaño del útero, placenta y el feto, entre otros. Estos factores n molestos para la mujer pues la limita en sus actividades diarias (2); también, su cotidianidad se ve afectada por los cambios emocionales y psicológicos, debido al estrés, la ansiedad y la depresión que algunas presentan (3).

Con respecto a dichos síntomas que pueden alterar su bienestar, es importante aclarar que las náuseas suelen presentarse en el primer trimestre de gestación, estas son esporádicas, matutinas pero no generan daño en la gestante; sin embargo, existen condiciones como la hiperémesis gravídica, con la cual esas náuseas y vómitos dejan de ser matutinas, se convierten en constantes generando pérdida de peso y deshidratación; por lo 
tanto, esta última si necesita atención hospitalaria de la mujer (4). Las náuseas matutinas y su incomodidad pueden manejarse con terapias alternativas como la aromaterapia. En el caso del dolor lumbar generado en el embarazo, se considera como una condición patológica de la cual se desconoce su etiología, siendo un fenómeno que ha tomado fuerza en su estudio e investigación, porque ha aumentado el número de mujeres que consultan a ginecólogos y traumatólogos para que les ayuden con su dolor; además, se considera como una afección que afecta la calidad de vida de la mujer y genera costos al sistema de salud (5). De ahí que sea importante por parte de la enfermería que aplique un abordaje holístico sobre este tipo de condiciones con terapias alternativas que ayuden a la mujer a tener una mejor gestación.

Por otro lado, es cierto que al producirse disconfort en la gestante, el personal de salud ha recurrido a controlarlo con fármacos, que, aunque son muy efectivos pueden tener efectos adversos. Un ejemplo de esto es el uso de la doxilamina + piridoxina muy efectiva para náuseas y vómito, pero que genera estados de somnolencia (6). Está claro que durante la gestación se debe tener mucho cuidado con el uso de medicamentos, según la FDA ( Food and Drug Administration) ya que algunos pueden causar daños en el feto (7). Esta es la razón por la cual la aromaterapia puede ser una excelente opción para proporcionar bienestar a la gestante y a su familia; es por eso, que su utilización puede contribuir en el cuidado más humanizado de la gestante, puesto que contribuye a disminuir dichos síntomas sin posibles efectos tanto en la mujer como en el feto. Se debe precisar que esta terapia no sólo alivia la condición física, sino que también contribuye al mejoramiento del estado emocional (8). La aromaterapia utiliza aceites esenciales hechos a base de semillas, tallos, hojas, pétalos, flores, cortezas, frutas, maderas, resinas, raíces, rizomas y hierbas, dando como resultado la obtención de varios casos positivos de mejora en pacientes que la utilizan (9).

Conviene subrayar que el uso de la aromaterapia se fundamenta en el reconocimiento de la persona como un ser holístico; dicha práctica le ofrece a las gestantes y a sus familias la oportunidad de ser comprendidos como seres importantes, en donde el único objetivo es ofrecerles confort y bienestar; en consecuencia, la aromaterapia proporciona relaciones e intervenciones desde la humanización en la práctica clínica con las gestantes, quienes desean vivir junto con su familia la experiencia más bonita y positiva. También es cierto que, para el manejo de muchas molestias generadas por los cambios durante el embarazo se aplican técnicas mecanicistas y rutinarias; no obstante, desde ya se debe tener en cuenta un paradigma constructivista, con una visión unitaria transformativa en la cual la enfermería, desde la empatía pueda contribuir a transformar las experiencias de las gestantes en positivas y enriquecedoras con el uso de técnicas sencillas, efectivas y sin efectos adversos (10).

Por tanto es necesario indicar que la aromaterapia es una rama de la medicina alternativa, que actualmente es recomendada a gestantes como terapia no farmacológica, disminuyendo el uso de medicamentos que puedan afectar la salud del embrión o feto. Además, se recomienda porque es fácil de emplear y promueve la salud física, emocional y psicológica (7). Se ha establecido que dichos aceites esenciales sirven para prevenir enfermedades y para recuperar la salud, promoviendo la integración del ser humano con el medio ambiente, a partir de un enfoque más humanizado y con una visión holística del ser humano; es decir, que la aromaterapia permite recuperar y armonizar el equilibrio del organismo. En este punto es importante aclarar que no todos los aceites esenciales pueden usarse durante la gestación, pues existen algunos que estimulan el músculo uterino (11) tales como: el cedro atlántico, la angélica, la aquilea o milenrama, el enebro, el hinojo, el laurel, la mejorana, el orégano, el romero, el tomillo y el toronjil (12). Sin embargo, los aceites esenciales de: lavanda, bergamota, nerolí, menta, limón, rosa, jengibre, entre otros, son seguros y proporcionan bienestar.

De acuerdo con lo planteado anteriormente, el objetivo de este estudio es identificar los beneficios que produce la aromaterapia en la gestación mediante una revisión documental.

\section{Metodología}

Se realizó una revisión descriptiva de estudios científicos consultados en bases de datos como: Science direct, google academic, EBSCO, Pubmed, Gale Power Search, BASE (Bielefeld academic search engine), Proquest. Esta búsqueda se efectuó sin límite de fecha debido a que es un fenómeno o tema de investigación que es poco estudiado en el área de la salud, pues se le considera como pseudociencia. Se examinaron artículos en idioma inglés, español y portugués. De 
Revista

Cienciavoluidado

Scientific Journal of Nursing

igual forma, se emplearon operadores booleanos como "AND " para conectar en las ecuaciones de búsqueda con los descriptores DECS: Aromatherapy, Pregnancy, Lavandula, Citrus, Ginger, Rosa. Como resultados se obtuvieron 50 artículos que cumplieron con el objetivo planteado, se seleccionaron aquellos que contenían información sobre el uso de la aromaterapia durante el embarazo. Para llevar a cabo este proceso, inicialmente se llevó a cabo la lectura del título y del abstract de todos los artículos localizados, permitiendo seleccionar 50 que cumplían con los criterios de inclusión y exclusión. Es necesario señalar que solamente se trabajó con artículos de investigación publicados en idioma español, inglés y portugués, sin importar fecha de publicación, pero que tuvieran como eje central el uso de la aromaterapia durante el embarazo. Así mismo, se excluyeron todos aquellos artículos que no indagaban sobre la utilidad de la aromaterapia durante el parto y el puerperio y otras técnicas de medicina alternativa o complementaria como la acupuntura, la fitoterapia, el reiki, entre otras. De los artículos que cumplían con el objetivo planteado, dos se encontraron en Sciencie direct, 14 en google academic, 19 en EBSCO, 5 en Pubmed, uno en Gale Power Search, seis en BASE y tres en Proquest. Igualmente, se localizaron 26 artículos que señalaban el trabajo realizado por estudios experimentales; otros seis fueron cuasiexperimentales, tres no experimentales, nueve hicieron parte de revisiones sistemáticas, cinco utilizaron metodología pre-experimental y un trabajo de grado.

Cabe resaltar que para hacer el análisis crítico de la literatura y confirmar la validez de cada publicación se utilizó el instrumento CASPe. Seguidamente se prosiguió a trabajar la información obtenida en una matriz de Microsoft Excel en donde se visibilizó la información en general, título, autores, objetivo, resumen, metodología, diseño, resultados y conclusión. Después de la lectura se pudieron obtener las siguientes categorías: 1. náuseas y vómitos durante el embarazo, 2. dolor en el embarazo, 3. estrés, fatiga y ansiedad, 4. sueño y picazón, 7. Manejo de presión arterial.

\section{Análisis}

Los resultados se clasificaron según la sintomatología que presentan las mujeres embarazadas durante la gestación y el uso de la aromaterapia para controlarlos, según los resultados encontrados en la literatura. Uso de aromaterapia para náuseas y vómitos
Ariadna María Angarita-Navarro, Paula Daniela Casas-Cárdenas, Jineth Paola López-Aguirre
Las náuseas y vómitos son signos y síntomas frecuentes durante la gestación; en tal sentido se han efectuado estudios que demuestran la efectividad de algunos aceites esenciales para controlar estos síntomas y generar bienestar.

Con relación a este tópico, en los artículos revisados se pudo evidenciar que el aceite esencial más utilizado para combatir las náuseas matutinas durante el embarazo es el de la menta, gracias a sus excelentes beneficios. Por otra parte, en un estudio de investigación en el que participaron 30 gestantes, el 17,5\% sintieron náuseas y vómitos, pero al usar el aceite esencial de menta, esta molestia disminuyó en un 8\% (13). En otra investigación de tipo experimental con una muestra de 66 gestantes, con la mitad de ellas se utilizó el aceite de menta, frente a la otra mitad o grupo control no se utilizó dicho aceite, demostrándose que hubo una leve reducción de las náuseas y vómitos en el grupo experimental (14). De igual forma, en otro estudio en el que también se utilizó el aceite esencial de menta, utilizando una muestra de 56 gestantes, donde 28 formaron parte del grupo control y 28 del experimental; al respecto se evidenció que el grupo experimental se mejoró. No obstante se le hacen críticas con respecto a los resultados que se obtuvieron del estudio, porque no hubo control en aspectos como: la edad, la raza, el nivel educativo y estado laboral de las participantes (15). Igualmente, en otra investigación de tipo experimental, los resultados demostraron que en el grupo experimental al cual se le aplicó el aceite esencial de menta, obtuvo mejoría en la primera semana, pero semanas después la sensación de náuseas fue igual tanto para el grupo control como para el experimental (16).

También se ha indagado en un estudio experimental sobre el uso del aceite esencial de menta combinado con el aceite de limón para evaluar su efecto en las náuseas y el vómito, demostrándose que ambos aceites funcionan al tercer día de uso; sin embargo, semanas después no fue significativa la diferencia comparada entre el grupo control y el experimental (17). En esta misma investigación, se usó la combinación del aceite esencial de menta con al aceite esencial de lavanda, durante las primeras semanas de gestación, probándose que los resultados fueron positivos y mostraron efectividad (18). De modo similar en otro estudio se empleó la combinación del aceite esencial de menta y el aceite esencial de jengibre, concluyéndose que en el grupo experimental hubo disminución de náuseas y vómito. Se debe pre- 
cisar que antes de usar la combinación de estos aceites esenciales, las gestantes tenían dichos malestares entre tres y cuatro veces al día, pero al comenzar a usarlos disminuyó a una sola vez al día (19).

De la misma forma el aceite esencial de menta tiene varias propiedades, razón por la cual está indicado como: acción antiinflamatoria, analgésico, antiinfeccioso, antimicrobiano, antiséptico, antiespasmódico, astringente, digestivo, carminativo, fungicida, estimulante nervioso, vasoconstrictor, descongestionante, y estomacal (20). Cabe destacar que sus propiedades para mejorar los síntomas gastrointestinales, es la razón por la cual se usa en gestantes, en virtud de que diferentes estudios han justificado su utilización para disminuir las náuseas durante el primer trimestre del embarazo, sin producir efectos adversos; es por eso que se le considera como una de las opciones para controlar tal sintomatología.

Igualmente se puede indicar que el aceite de limón también es muy usado debido a su facilidad para encontrarlo y prepararlo. Desde este punto de vista, en un estudio realizado por Rofi'ah (21) se demostró que al realizar aromaterapia con aceite esencial de limón se logra reducir la emesis gravídica, evitando el uso de medicamentos (21). También se sabe que las náuseas y vómitos tienden a presentarse en el primer trimestre y ceden al terminar la semana 13; de ahí que un estudio realizado por Wiulin (22) se demuestra la efectividad del aceite de limón durante este periodo; además en esta investigación se midieron las náuseas y vómito antes y después de utilizar dicho aceite esencial, resultando efectivo su uso como aromaterapia (22).

Lo expuesto anteriormente se puede justificar con un estudio de Pitriani, quien demuestra cómo una gestante al consultar a un servicio indicaba que durante las primeras semanas de gestación no podía comer, que tenía náuseas y vómitos dos veces al día, y que al utilizar con ella el aceite esencial de limón, se obtuvo como resultado que sintiera mejoría permitiéndole comer sus alimentos normalmente (23). Este estudio se puede comparar con el realizado en un centro de salud de Teling Atas, exactamente en Wanea en donde se efectuó una investigación con la que se quería conocer el comportamiento del aceite de limón en las gestantes para controlar sus náuseas y vómitos; dicho estudio concluyó que existe eficacia y le proporciona a la mujer biene- star (24). El mismo resultado se obtuvo en la investigación de Kia y compañeros en el año 2014, quienes también demostraron que el uso del aceite de limón era efectivo para curar dichas molestias (25).

Se puede mencionar que en otro artículo se evidenció que la utilización de terapias con ayuda de la aromaterapia con aceite esencial de limón y la acupresión para la disminución de las náuseas y vómito, dio como resultado que la combinación de estas técnicas alternativas disminuye significativamente dichos síntomas en las primeras semanas de gestación (26).

La anterior información encontrada en los diferentes artículos permite reconocer la efectividad del aceite esencial de limón para controlar las náuseas y el vómito en las primeras semanas de gestación (27-29).

Así mismo, del aceite esencial de limón, se debe agregar que tiene propiedades so antisépticas, astringentes, desintoxicantes, refuerza el sistema inmunológico, acelera la producción de glóbulos blancos; de igual manera es útil para contrarrestar la acidez y las úlceras mejorando la digestión, pues contribuye a mejorar el carbonato y bicarbonatos de potasio y calcio a nivel gastrointestinal, siendo muy eficaz para controlar náuseas y vómitos (20). Otro rasgo a tener en cuenta que este aceite esencial desde la perspectiva emocional sirve para mejorar los estados de ánimo. Entonces, estos efectos o propiedades en el sistema gastrointestinal se tienen en cuenta para mejorar malestares en las gestantes a causa de las náuseas y vómitos, convirtiéndose así en otro aliado; razón por la cual se invita a usarlo por su eficacia, sin altos costos económicos y sin efectos adversos.

Por otro lado, así como en el caso de la menta, que se ha comprobado su efectividad para las náuseas y vómito, se han realizado estudios en los cuales se combinan el aceite de limón con otros aceites esenciales (30). Es el caso de la combinación del aceite de jengibre y limón que muestran altos beneficios para tales molestias en el primer trimestre de gestación (19). Otro estudio que corroboró la efectividad sobre la combinación de aceites esenciales fue el realizado por los investigadores Sarwinanti e Istiqomah, quienes experimentaron con la combinación de aceite de limón y lavanda, con exitosos resultados para las gestantes (31). En el 2018 realizaron otro estudio sin tener en cuenta el 


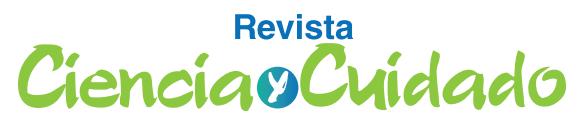

Scientific Journal of Nursing

aceite de limón, utilizando solamente la combinación de aceite de lavanda y el jengibre para las náuseas y vómito, concluyendo que es más efectivo el aceite de lavanda para esta sintomatología en la gestante (32).

Por último, se puede señalar que además de la utilización de los aceites esenciales como la menta, el jengibre, la lavanda y el limón en combinación (33), incluso se ha determinado que el uso del aceite esencial de jengibre también funciona para disminuir las náuseas y el vómito durante la gestación (34-35). Se debe agregar que otro aceite esencial utilizado para controlar dicha sintomatología en el embarazo debido a sus cambios normales es el de cajeput, el cual e ayuda a disminuir la frecuencia de las náuseas en el primer trimestre (36).

En definitiva, se puede concluir que el uso de combinaciones de aceites esenciales, son una buena técnica para potencializar sus propiedades y beneficiar a la gestante. Entonces, se recomienda que, en la consulta de control prenatal y en la interacción que se tiene en los cursos de preparación para la maternidad y paternidad, se deben propiciar espacios para escuchar a las gestantes y sus familiares. Simultáneamente se les debe explicar que si experimentan náuseas y vómitos, se pueden utilizar los aceites esenciales, indicándoles la intención de su uso y cómo aplicarlos, expresándoles que no tendrán ningún efecto en sus hijos por nacer y recomendarles su uso en casa de una forma controlada. Los aceites para recomendar son: el aceite esencial de menta, el aceite esencial de limón, el aceite esencial de jengibre; además de combinaciones como: aceite esencial de jengibre + aceite esencial de limón; aceite esencial de limón + aceite esencial de lavanda; aceite esencial de menta + aceite esencial de limón; aceite esencial de menta + aceite esencial de lavanda; aceite esencial de menta + aceite esencial de jengibre. Su uso puede ser inhalado, ya sea por difusor aplicando dos gotas de cada aceite esencial, o con un pañuelo, aplicando dos gotas de cada aceite esencial (puede ser uno solo o lograr combinarlos bajo las recomendaciones dadas) y se acercan a la nariz por aproximadamente 5- 10 minutos haciendo respiraciones lentas y profundas.

Uso de la aromaterapia para el manejo del dolor durante la gestación

Dentro de los artículos encontrados se evidenció que la aromaterapia también se usa para controlar algunos dolores que se presentan en la gestación. El dolor lumbar
Ariadna María Angarita-Navarro, Paula Daniela Casas-Cárdenas, Jineth Paola López-Aguirre

es una molestia frecuente principalmente en el último trimestre del embarazo y se debe a los cambios generados a nivel físico en el cuerpo de la gestante. Ante lo incómodo que puede ser este dolor, algunas pacientes han optado por utilizar no sólo fármacos para calmarlo, sino que el 25\% se han aplicado técnicas complementarias, siendo la aromaterapia una de las más usadas (37). Por otro lado, se ha identificado el efecto del aceite esencial de rosa damascena comparado con placebos en un grupo control, logrando disminuir los dolores lumbres (38). Asimismo, se encontró en un artículo los resultados sobre la utilización del aceite de coco (como aceite base) y aceite esencial de lavanda para realizar masajes lumbares en mujeres gestantes que presentan dolor, demostrando que es positiva su aplicación al disminuir los dolores lumbares durante el tratamiento (39).

Por tal razón, es importante indicar que durante el embarazo existen otros tipos de dolores que se pueden presentar como contracciones de braxton hicks. De allí que en una investigación se quiso demostrar la efectividad del aceite esencial de lavanda para controlar dichos dolores debido a las contracciones, infiriendo que las gestantes del grupo experimental quienes estuvieron expuestas a este aceite por 20 minutos tuvieron efectos positivos y el control del dolor fue bueno (40).

De igual forma, en la búsqueda se encontró un artículo que tenía como objetivo determinar la efectividad de la aromaterapia en el procedimiento de amniocentesis; en este punto es necesario aclarar que este es un procedimiento que sólo se lleva a cabo en algunos casos para efectuar diagnósticos de enfermedades genéticas, infecciosas, entre otras; por tanto, no es un examen de rutina. En el estudio se probó el aceite esencial de mentol para disminuir la ansiedad y el dolor, sin embargo, los resultados fueron desalentadores, puesto que no funciona para dichos fines (41).

Con referencia al aceite de lavanda que químicamente contiene linalol y acetato de linalilo, compuestos que se absorben fácilmente por la piel, llegando al sistema límbico del cerebro, logrando depresión en el sistema nervioso central; esto se logra efectuando masajes con gotas del aceite esencial de lavanda en aceite transportador como el aceite mineral o el aceite de almendras, prosiguiéndose a realzar el masaje en la región lumbar de la mujer. En la gestante se puede utilizar esta téc- 
nica, ya que puede ser muy relajante, pues logra disminuir la ansiedad, el temor, fluctuaciones emocionales negativas y también el dolor lumbar a causa del aumento del peso en su cuerpo. Las propiedades específicas del aceite de lavanda son: manejo de quemaduras en la piel, estrés, dolor de cabeza, disminuye dolores musculares y para mejorar el sistema inmunológico (20). Además de la recomendación de su uso en masajes para el dolor lumbar, también se puede emplear inhalado con un efecto principalmente relajante.

Uso de la aromaterapia para manejo del estrés, ansiedad y fatiga

Se ha mencionado anteriormente que, en el transcurso de toda la gestación también hay cambios psicológicos y emocionales los cuales pueden ser controlados con la aromaterapia porque ayuda a mejorar el sistema nervioso autónomo (42).

En un estudio realizado por Aisyah se demuestra cómo el uso del aceite de lavanda en conjunto con la musicoterapia lograron disminuir el nivel de ansiedad en las mujeres gestantes (43). Otro estudio que refleja las propiedades de la lavanda es el de Bastard y Tiran, quienes comprueban la gran efectividad para tratar la ansiedad, el estrés y la depresión durante el embarazo (44); de igual forma, lo menciona Sasirekha en su investigación, probando el uso del aceite de lavanda para contrarrestar la ansiedad (45). Con respecto al estrés que se puede generar durante la gestación, no propiamente por dicha condición, sino también por factores externos, se ha probado el beneficio del aceite esencial de lavanda (46-48).

Por último, otro de los problemas que se presentan durante la gestación es la fatiga; ante esta situación, en un artículo publicado en el año 2020 se menciona que la intervención por medio del ejercicio y la aromaterapia en las gestantes han facilitado la disminución de la fatiga (49).

\section{Uso de la aromaterapia para manejo de sueño}

Durante el embarazo, los ciclos de luz - oscuridad son períodos que están relacionados con la secreción de hormonas como: la melatonina, la hormona de crecimiento, el cortisol y la hormona estimulante de la tiroides, por tanto, cualquier afectación en este ciclo de sueño puede influir en el feto; no obstante, se ha con- firmado que, durante la gestación, la mujer tiene dificultades para conciliar el sueño en el último trimestre del embarazo (50). Esta es la razón por la cual se debe acompañar a la mujer y conocer cómo son sus ciclos de sueño durante el embarazo, y ayudarla si es necesario. La aromaterapia es una técnica que puede contribuir a mejorar esta condición. Según Samadi en su publicación del año 2021 indica que el aceite de lavanda fue estudiado experimentalmente en gestantes para determinar su efectividad con respecto al sueño, obteniendo como resultado que tal aceite esencial logra mejorar la calidad del sueño durante el embarazo (51).

Como se puede deducir en los resultados de las diferentes investigaciones se evidenció que el aceite esencial de lavanda fue el más utilizado, con excelentes resultados. Como se especificó anteriormente, este aceite al tener un efecto depresor en el sistema nervioso, permite relajarse, disminuir la ansiedad y conciliar el sueño fácilmente. Sin embargo, aunque en los artículos revisados se menciona principalmente la lavanda, se sabe que existen otros aceites esenciales que también se pueden utilizar para relajar y mejorar estados de ánimo, como lo son: la mandarina, el limón, la bergamota y la naranja (20).

\section{Uso de la aromaterapia para manejo del prurito}

El prurito es un síntoma que no se presenta de forma universal durante el embarazo; no obstante, algunas gestantes pueden tener cierta molestia por varias causas como: candidiasis, hepatitis vírica o tóxica, hemólisis, policitemia vera, esclerosis múltiple, en casos de hiper o hipotiroidismo, entre otras (52). Para estos casos la aromaterapia puede ser una solución a tal incomodidad en la mujer y según Asih en su publicación del año 2021, expresa que este tipo de enfermedades se pueden tratar con la aromaterapia (53).

Por tanto se recomienda el uso del aceite esencial de menta inhalado, ya sea mediante un pañuelo o un difusor.

Ventajas y desventajas de la aromaterapia durante la gestación

A pesar de que los estudios han demostrado la efectividad de la aromaterapia para el manejo y el control de signos y síntomas molestos durante el embarazo, sin embargo se han encontrado publicaciones en don- 


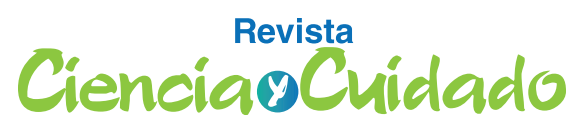

Scientific Journal of Nursing

de se indica que dicha terapia debe ser manejada por expertos, pues según Sibbritt (2014), las mujeres que se autoadministraban aromaterapia sin orientación de un profesional presentaron reacciones alérgicas y fiebre ante el uso de algunos aceites esenciales (54). Definitivamente hay que hacer un llamado a los profesionales de la salud para que se familiaricen con las propiedades de los aceites esenciales y así poder orientar adecuadamente a las gestantes. No obstante, se debe saber que existen aceites esenciales que aumentan las contracciones uterinas o que pueden tener efectos en el sistema nervioso central del feto, como el aceite esencial de zanahoria, orégano y zadoaria provocando abortos o amenazas de parto pretérmino (55).

Conviene subrayar que la información anterior no permite afirmar que los aceites esenciales sean dañinos en la gestación, solo hay que conocer los efectos sobre la aromaterapia, y cuando sea necesario recomendarla como técnica para proporcionar a la gestante salud y bienestar (56). Como se ha dicho, en realidad son más los beneficios de la aromaterapia que las mismas desventajas (57-59); al respecto testimonios de gestantes en una investigación realizada en el año 1994 indicaron que sintieron un efecto positivo en su salud física y psicológica (60).

\section{Conclusiones}

El uso de aromaterapia en la gestación proporciona a la mujer importantes beneficios, disminuyendo síntomas molestos que se pueden presentar por cambios nor- males durante el embarazo a nivel psicológico y anatomofisiológico. Al efectuar la revisión bibliográfica se encontraron artículos indicando que los aceites esenciales como: la lavanda, el limón, la menta, la naranja, la rosa damascena, el té y el jengibre, entre otros, tienen propiedades que generan en el cuerpo diferentes acciones y reacciones que proporcionan bienestar. Al clasificar los resultados se pudo evidenciar que los síntomas que genera mucha más molestia en las gestantes son las náuseas y vómitos, existiendo aceites esenciales que logran controlarlos y disminuir su frecuencia. Por otra parte, se encontró que en la mayoría de los artículos examinados se concluye sobre la importancia de la aplicación en mujeres gestantes de la aromaterapia; sin embargo, en algunos se especifica que algunas mujeres reaccionaron negativamente, al no existir acompañamiento por parte de un conocedor de la terapia, sobretodo al usar algunos aceites que no pueden tener contacto directo con la piel y que deben usarse con un aceite base, lo que les produjo alergias e irritación. Por último, se concluye que las terapias complementarias y/o integrativas como la aromaterapia tienen resultados positivos y ayudan a las mujeres gestantes a pasar por esta etapa de una forma significativa, teniendo una mejor calidad de vida, centrándose en la aromaterapia como una técnica indolora y eficaz.

\section{Conflicto de interes}

Los autores declaran no tener ningun conflicto de intereses

\section{Referencias Bibliográficas}

1. Gómez-Vilches A, Celesia MC, Fernández CL. Cambios anatómicos y fisiológicos del embarazo: sus implicancias anestesiológicas. 2000; 339-44.

2. Matthews A, Haas DM, O’Mathúna DP, Dowswell T. Interventions for nausea and vomiting in early pregnancy. Cochrane Database of Systematic Reviews. 2015;2015(9).

3. Figuero-Ruiz E, Prieto-Prieto I, Bascones-Martínez A. Cambios hormonales asociados al embarazo: Afectación gingivo-periodontal. Avances en Periodoncia e Implantología Oral. 2006;18(2):101-13.

4. González-gonzález A, Álvarez-silvares E, Veiga-vázquez A. Síntomas y signos digestivos durante la gestación: náuseas y vómitos / hiperemesis gravídica. 2011;37(10).

5. Munjin M, Ilabaca F, Rojas J. Dolor lumbar relacionado al embarazo. 2007;258-65.

6. Ministerio Sanidad P social e igualdad. FICHA TÉCNICA: Doxilamina succinato+ Piridoxina hidrocloruro. Agencia Española de Medicamentos y productos sanitarios [Internet]. 2020;4(Día 2):12-26. Available from: 
Scientific Journal of Nursing

https://cima.aemps.es/cima/pdfs/es/ft/44139/FT_44139.pdf

7. Khresheh R. How women manage nausea and vomiting during pregnancy: A Jordanian study. Midwifery [Internet]. 2011;27(1):42-5. Available from: http://dx.doi.org/10.1016/j.midw.2009.12.002

8. Bastard J, Tiran D. Aromatherapy and massage for antenatal anxiety: Its effect on the fetus. Complementary Therapies in Clinical Practice. 2006;12(1):48-54.

9. Farrar AJ, Farrar FC. Clinical Aromatherapy. Nursing Clinics of North America. 2020;55(4):489-504.

10. Prieto Bocanegra BM, Gil Sosa JC, Madrid Simbaqueba DC. Terapias complementarias durante la gestación y parto. Revisión integrativa. Revista Cuidarte. 2020;11(2):1-14.

11. Artuz MA, Restrepo H. Colombia Médica Colombia M é dica. 2002;33:65-71.

12. Lomazzi G. Aromaterapia: antiguos secretos de los aceites esenciales que ayudan a recuperar la salud del cuerpo y del alma. BIenestar. Bogotá; 2008.

13. Putri SS, Isnaini N, Yuviska IA. The effect of mint oil on nausea and vomiting during pregnancy. 2020;03(1):359.

14. Amzajerdi AA, Keshavarz M, Montazeri A. Efecto del aroma de menta sobre las náuseas, los vómitos y la ansiedad en mujeres embarazadas . 2019;

15. Joulaeerad N, Ozgoli G, Hajimehdipoor H, Ghasemi E, Salehimoghaddam F. Effect of aromatherapy with peppermint oil on the severity of nausea and vomiting in pregnancy: A single-blind, randomized, placebo-controlled trial. Journal of Reproduction and Infertility. 2018;19(1):32-8.

16. Pasha H, Behmanesh F, Mohsenzadeh F, Hajahmadi M, Moghadamnia AA. Study of the effect of mint oil on nausea and vomiting during pregnancy. Iranian Red Crescent Medical Journal. 2012;14(11):744-7.

17. Safajou F, Soltani N, Taghizadeh M, Amouzeshi Z, Sandrous M. The effect of combined inhalation aromatherapy with lemon and peppermint on nausea and vomiting of pregnancy: A double-blind, randomized clinical trial. Iranian Journal of Nursing and Midwifery Research. 2020;25(5):401-6.

18. Mahmoud R, Ghani A, Tawfik A, Ibrahim A. The Effect of Aromatherapy Inhalation on Nausea and Vomiting in Early Pregnancy: A Pilot Randomized Controlled Trial. 2013;3(6):10-22.

19. Kustriyanti, D. Adityasari Putri A. The Effect of Ginger and Lemon Aromatherapy on Nausea and Vomiting among Pregnant Women. Jurnal Keperawatan Soedirman [Internet]. 2019;14(1):15-22. Available from: http://proxy.kc.edu/login?url=https://search.ebscohost.com/login.aspx?direct=true\&db=edsdoj\&AN=edsdoj. fc608a95f4b6d979fe6f3893a9155\&site=eds-live. Diakses 23 Februari 2021

20. Ali B, Al-Wabel NA, Shams S, Ahamad A, Khan SA, Anwar F. Essential oils used in aromatherapy: A systemic review. Asian Pacific Journal of Tropical Biomedicine [Internet]. 2015;5(8):601-11. Available from: http:// dx.doi.org/10.1016/j.apjtb.2015.05.007

21. Rofi'ah S, Widatiningsih S, Sukini T. Efektivitas Aromaterapi Lemon untuk Mengatasi Emesis Gravidarum. Jurnal Kebidanan. 2019;9(1):9-16.

22. Wiulin Setiowati dan Nor Aida Arianti. Pengaruh Pemberian Aromaterapi Lemon (Citrus Lemon) Terhadap Mual Muntah Pada Ibu Hamil Trimester I. Jurnal Darul Azhar [Internet]. 2019;7(1):77-82. https://jurnal-kesehatan.id/index.php/JDAB/. Available from: https://jurnal-kesehatan.id/index.php/JDAB/article/view/132

23. Pitriani R, Hang S, Pekanbaru T, Mustafa J, No S, Pos K. Lemon Aroma Therapy For Overcoming Nausea Vomiting At first Trimester Pregnancy Clinic Primary Afiyah In Pekanbaru. 2019;2(1):126-9.

24. Purwandari A, Tuju S, Lumy F, Anisa W. Lemon Suangi (Citrus limon) Aromatherapy to Reduce Vomitting on Early First Trimester Pregnancy at Teling Public Health Center Wanea District Manado City. Parity. 
2019;30(1):6.

25. Kia PY, Safajou F, Shahnazi M, Nazemiyeh H. The effect of lemon inhalation aromatherapy on nausea and vomiting of pregnancy: A double-blinded, randomized, controlled clinical trial. Iranian Red Crescent Medical Journal. 2014;16(3).

26. Magfirah M, Fatma S, Idwar I. The effectiveness of acupressure therapy and aromatherapy of lemon on the ability of coping and emesis gravidarum in trimester i pregnant women at langsa city community health centre, aceh, indonesia. Open Access Macedonian Journal of Medical Sciences. 2020;8(E):188-92.

27. Cholifah S, Nuriyanah TE. Aromaterapi Lemon Menurunkan Mual Muntah pada Ibu Hamil Trimester I. Jurnal Kebidanan Midwiferia. 2019;4(1):36.

28. Nurulicha, Aisyah S. the Influence of Lemon Inhalation on Reduction of. 2019;8(1):157-65.

29. Oktavia NS, Susanti D, Anggalia R. the Effect of Lemon Tea Aromatheraphy on Emesis. Jurnal Kesehatan Ibu dan Anak. 2018;12(2):145-52.

30. Sulistiarini U, Widyawati MN, Rahayu DL. Studi Literatur: Acupressure Pericardium Dan Aromatherapy Citrus Untuk Mengurangi Mual Muntah Ibu Hamil. Jurnal Kebidanan. 2018;8(2): 146.

31. Sarwinanti S, Istiqomah NA. Perbedaan aromatherapi lavender dan lemon untuk menurunkan mual muntah ibu hamil. Jurnal Kebidanan dan Keperawatan Aisyiyah. 2020;15(2):185-95.

32. Rahayu R, Sugita S. Efektivitas Pemberian Aromaterapi Lavender Dan Jahe Terhadap Penurunan Frekuensi Mual Muntah Pada Ibu Hamil Trimester I Di BPM Trucuk Klaten. Jurnal Kebidanan dan Kesehatan Tradisional. 2018;3(1):19-26.

33. Fattah A, Hesarinejad Z, Gharaii NR, Nasibi M. The effect of aromatherapy on nausea and vomiting during pregnancy: a systematic review and meta-analysis. International Journal of Pediatrics [Internet]. 2019;7(63):906170. Available from: http://eds.b.ebscohost.com/eds/detail/detail?vid=0\&sid=fldb59cc-1097-49ba-9281-aa49fafbd906\%40pdc-V-sessmgr03\&bdata=Jmxhbmc9dHImc210ZT11ZHMtbG12ZQ\%3D\%3D\#AN=edsdoj.03a074b43c7747b39158ce1b858fc9af\&db=edsdoj

34. Dewianti NM, Witari NND. Effectiveness of ginger aromatherapy towards nausea and vomiting on first trimester pregnant women at BPM (private practice midwives) Ni Wayan Suri in Denpasar City. International Journal of Research in Medical Sciences. 2019;7(2):347.

35. Wirda W, Ernawati E, Oktaviana D, Suardi S, Nofia N. Pengaruh Pemberian Aromaterapi Jahe Terhadap Penurunan Emesis Gravidarum Pada Ibu Hamil Trimester Pertama Di Wilayah Kerja Puskesmas Mangarabombang Kabupaten Takalar. Journal of Islamic Nursing. 2020;5(2):127.

36. Kusparlina EP. 52| Publisher: Humanistic Network for Science and Technology Aromatherapy Cajuput Oil for Emesis Gravidarum. Health Notions [Internet]. 2019;3(1):52-6. Available from: http://heanoti.com/index. $\mathrm{php/hnhttp://heanoti.com/index.php/hn/article/view/hn301210}$

37. Hughes CM, Liddle SD, Sinclair M, McCullough JEM. The use of complementary and alternative medicine (CAM) for pregnancy related low back and/ or pelvic girdle pain: An online survey. Complementary Therapies in Clinical Practice [Internet]. 2018;31:379-83. Available from: https://doi.org/10.1016/j.ctcp.2018.01.015

38. Shirazi M, Mohebitabar S, Bioos S, Yekaninejad MS, Rahimi R, Shahpiri Z, et al. The Effect of Topical Rosa damascena (Rose) Oil on Pregnancy-Related Low Back Pain: A Randomized Controlled Clinical Trial. Journal of Evidence-Based Complementary and Alternative Medicine. 2017;22(1):120-6.

39. Amellia, S. W. N., \& Utami RW. The Benefits of Prenatal Aromatherapy Massage and Prenatal Yoga in Improving Beta Endorphin and Alleviating Back Pain for Pregnant Women. 2021;34(Ahms 2020):15-9.

40. Christiana A, P TU. LAVANDULA OFFICINALIS PARA LA AROMATERAPIA CON TÉCNICAS DE BO- 


\section{LOS Y SUS EFECTOS SOBRE LA DISMINUCIÓN DEL EMBARAZO EN LOS TRIMESTRES II Y III Ari} Christiana, Tri Utami P. :11-8.

41. Hanprasertpong T, Kor-Anantakul O, Leetanaporn R, Suwanrath C, Suntharasaj T, Pruksanusak N, et al. Reducing pain and anxiety during second trimester genetic amniocentesis using aromatic therapy: A randomized trial. Journal of the Medical Association of Thailand. 2015;98(8):734-8.

42. Igarashi T. Physical and psychologic effects of aromatherapy inhalation on pregnant women: A randomized controlled trial. Journal of Alternative and Complementary Medicine. 2013;19(10):805-10.

43. Aisyah RD, Suparni S, Fitriyani F. Effect of Combination of Aroma Therapy Lavender and Classical Music Towards a Decrease in Anxiety Levels Moderate and Severe on Third Trimester Pregnant Women. Strada Jurnal Ilmiah Kesehatan. 2019;8(2):117-22.

44. Bastard J, Tiran D. Reprint of: Aromatherapy and massage for antenatal anxiety: Its effect on the fetus. Complementary Therapies in Clinical Practice [Internet]. 2009;15(4):230-3. Available from: http://dx.doi. org/10.1016/j.ctcp.2009.06.010

45. Sasirekha C. A quasi experimental study to assess the effectiveness of aromatherapy on the level of anxiety among primi mothers in selected maternity Hospitals at Dindigul District. 2015; Available from: http://repository-tnmgrmu.ac.in/id/eprint/776

46. Chen P, Chou C, Yang L, Tsai Y, Chang Y, Liaw J, et al. Un ensayo controlado aleatorio, prospectivo, longitudinal. 2017.

47. Primawati AS, Widyawati MN, Admini A. Penurunan Tingkat Stres Ibu Hamil Dengan Terapi Musik Dan Aromatherapy Pada Kelas Ibu Hamil. Jurnal Kebidanan. 2018;8(1):37.

48. Igarashi, T. Fujita M. Effects of aromatherapy for self-care during pregnancy. Departaments of maternity nursing and midwifery; 2010. p. 101-12.

49. Kartilah T, Februanti S. Effectiveness of progressive muscle relaxation and aromatherapy on fatigue in pregnant mothers. Medisains. 2020;18(1):14.

50. Ayala-Moreno, M.R. Velázquez-Martínez, R. Melgarejo-Gutiérrez, M. González-Méndez, C. Estrada-Ramírez, E. Vergara-Castañeda A. Papel de las alteraciones del sueño durante la gestación en la programación del feto para el desarrollo de obesidad y enfermedades crónicas degenerativas. Gaceta Medica de Mexico. 2019;155(4):417-22.

51. Samadi Z, Jannati Y, Hamidia A, Mohammadpour RA, Hesamzadeh A. The effect of aromatherapy with lavender essential oil on sleep quality in patients with major depression. Journal of Nursing and Midwifery Sciences. 2021;8(2):67-73.

52. Sánchez-Méndez JI, López-Rodríguez MJ. Manejo del prurito durante el embarazo. MEDIFAM - Revista de Medicina Familiar y Comunitaria. 2001;11(4):187-93.

53. Asih FR, Husin F, Suwarsa O, Fidrianny I, Hilmanto D. A randomized controlled trial of combination of peppermint, lavender, and turmeric oil for antipruritic agent in pregnant women. Medical Journal of Indonesia. 2021;30(1):39-44.

54. Sibbritt DW, Catling CJ, Adams J, Shaw AJ, Homer CSE. The self-prescribed use of aromatherapy oils by pregnant women. Women and Birth [Internet]. 2014;27(1):41-5. Available from: http://dx.doi.org/10.1016/j. wombi.2013.09.005

55. Dosoky NS, Setzer WN. Maternal reproductive toxicity of some essential oils and their constituents. International Journal of Molecular Sciences. 2021;22(5):1-31.

56. Buckle J, Ryan K CKB. Clinical Aromatherapy for Pregnancy, Labor and Postpartum. International Journal 
Scientific Journal of Nursing

of Childbirth Education. 2014;10;29(4):21-7.

57. Williams W. Preconception care and aromatherapy in pregnancy. The international journal of clinical aromatherapy. 2005;2(1):15-9.

58. López EA, Pérez JH, Ramos EM. Evendencia cientifíca sobre el uso del aceite de rosa mosqueta en el embarazo: Una revisió de la bibliografía. Medicina Naturista. 2013;7(2):94-8.

59. Navaee M, Rakhshkhorshid M. Comparing the Effect of Foot Massage with Grape Seed Oil and Sweet Almond Oil on Physiological Leg Edema in Primigravidae: A Randomized Clinical Trial. Evidence-based Complementary and Alternative Medicine. 2020;2020.

60. Saitoh I. A Study of Aromatherapy for Pregnant Women. Olfaction and Taste XI. 1994;336-7. 